\title{
Mitochondrial Abnormalities in Alzheimer Disease
}

\author{
George Perry ${ }^{1,2}$, Xinglong Wang ${ }^{2}$, Mark A. Smith ${ }^{2}$, Xiongwei Zhu ${ }^{2}$ \\ ${ }^{1}$ College of Sciences, University of Texas at San Antonio, San Antonio, TX 78249 \\ ${ }^{2}$ Case Western Reserve University, Cleveland, $\mathrm{OH} 44106$
}

Alzheimer's disease (AD) is the most prevalent form of dementia in the elderly. Mitochondrial dysfunction is a prominent and early feature of $\mathrm{AD}[1]$, although the underlying mechanism is still not clear. Recent studies demonstrate that mitochondria are dynamic organelles that undergo continual fission and fusion events which regulate the morphology and distribution of mitochondria, and also serve crucial physiological function[2]. Although an impaired balance of mitochondrial fission and fusion is implicated in neurodegenerative diseases including Charcot-Marie-Tooth neuropathy type 2A and optic neuropathy, its involvement in the pathogenesis of $\mathrm{AD}$ has not been investigated. The purpose of our current study is to understand whether mitochondrial dynamics and distribution is involved in mitochondrial dysfunction and neuronal dysfunction in AD.

In an effort to characterize specific cytological abnormalities in mitochondria in vulnerable neurons, we performed morphometric ultrastructural analysis of biopsy specimens (to avoid agonal state) [3]. In the cytoplasm of pyramidal neurons, morphometric analysis showed that the area of intact mitochondria is significantly decreased in AD while there is no difference between the area of damaged mitochondria in AD or control cases. Since mitochondria are highly susceptible to morphological artifacts such as broken cristae, through inadequate fixation, that controls and AD cases showed similar frequency of mitochondria with broken cristae suggests that fixation was similar for each group. Neurons in the AD cases containing NFT showed no significant differences in mitochondria compared to others. Surprisingly, we found a small but significant reduction in mitochondria number in AD. Additionally, the average size of mitochondria was significantly enlarged in AD vulnerable neurons, suggesting a disturbance in mitochondrial morphology control. Indeed, we found that the levels of DLP1, OPA1, Mfn1 and Mfn2C were significantly decreased, yet levels of Fis1 were significantly increased, in AD hippocampal tissues compared to age-matched controls by immunoblot analysis [4]. Interestingly, although all these proteins demonstrate even distribution in the cytoplasm and processes of pyramidal neurons in age-matched control hippocampus, they appeared to accumulate in the soma but not in the processes of pyramidal neurons in $\mathrm{AD}$ hippocampus. Given that OPA1, Fis1, and Mfn1/2 are all mitochondrial membrane proteins, the changes in their distribution to soma in AD neurons, suggest changes in mitochondria distribution in these neurons. Indeed, our ultrastructural studies demonstrated altered mitochondrial distribution in pyramidal neurons in AD brain with more mitochondria being distributed in the proximal area than in distal area in the cytoplasm compared to age-matched control brain and a decreased mitochondrial coverage in axons.

We next explored whether changes in the expression of mitochondrial fission/fusion proteins cause abnormal mitochondrial morphology and distribution in AD neurons in cell models [4]. The expression of these proteins was manipulated in M17 cells and primary hippocampal neurons in a way that mimicked their expression changes in AD (i.e., reduced expression of DLP1, OPA1, Mfn $1 / 2$ or increased expression of Fis1). Although these manipulations caused different effects on mitochondrial morphology, they all caused reduced mitochondrial density in the cell periphery (M17 cells) or neuronal processes (primary neurons) which correlated with reduced spine numbers (primary neurons), 
suggesting that the altered expression of these proteins may play an important role in mitochondrial redistribution and synaptic dysfunction in AD neurons.

To pursue the potential cause of such abnormal mitochondrial dynamics in AD neurons, we investigated the effect of A $\beta$ PP overexpression and soluble $\mathrm{A} \beta$ oligomers on mitochondrial dynamics in cultured neurons [4,5]. These manipulations caused reduced expression of DLP1 and OPA1 while increased expression of Fis1, consistent with our findings in AD brains. Importantly, confocal and electron microscopic analysis demonstrated that $\mathrm{A} \beta \mathrm{PP}$ oeverexpression, likely through the increased production of $A \beta$ (Figure 1), caused mitochondrial fragmentation and reduced mitochondrial coverage in neuronal processes similar to the mitochondrial abnormalities found in AD brain.

Overall, we demonstrated abnormal mitochondrial dynamics and distribution in AD cell models and in susceptible neurons in the brain from $\mathrm{AD}$ patients and suggest that an altered balance in mitochondrial fission and fusion is likely an important mechanism leading to mitochondrial and neuronal dysfunction in $\mathrm{AD}$ brain. As such, the modulation of mitochondrial dynamics may prove to be a valuable therapeutic target of AD.

\section{References:}

1. $\quad$ Castellani R, et al. J Neurosci Res. 70(3) (2002) 357-60.

2. Detmer SA, et al. Nat Rev Mol Cell Biol. 8(11) (2007) 870-9.

3. $\quad$ Hirai K, et al. J Neurosci. 21(9) (2001)3017-23

4. $\quad$ Wang X, et al. J Neurosci. 29(28) (2009) 9090-9103

5. $\quad$ Wang X, et al. Proc Natl Acad Sci U S A. 105(49) (2008)19318-23

6. This research was supported by the Alzheimer's Association (IIRG-10-173358) and NIH (R01 AG031852).

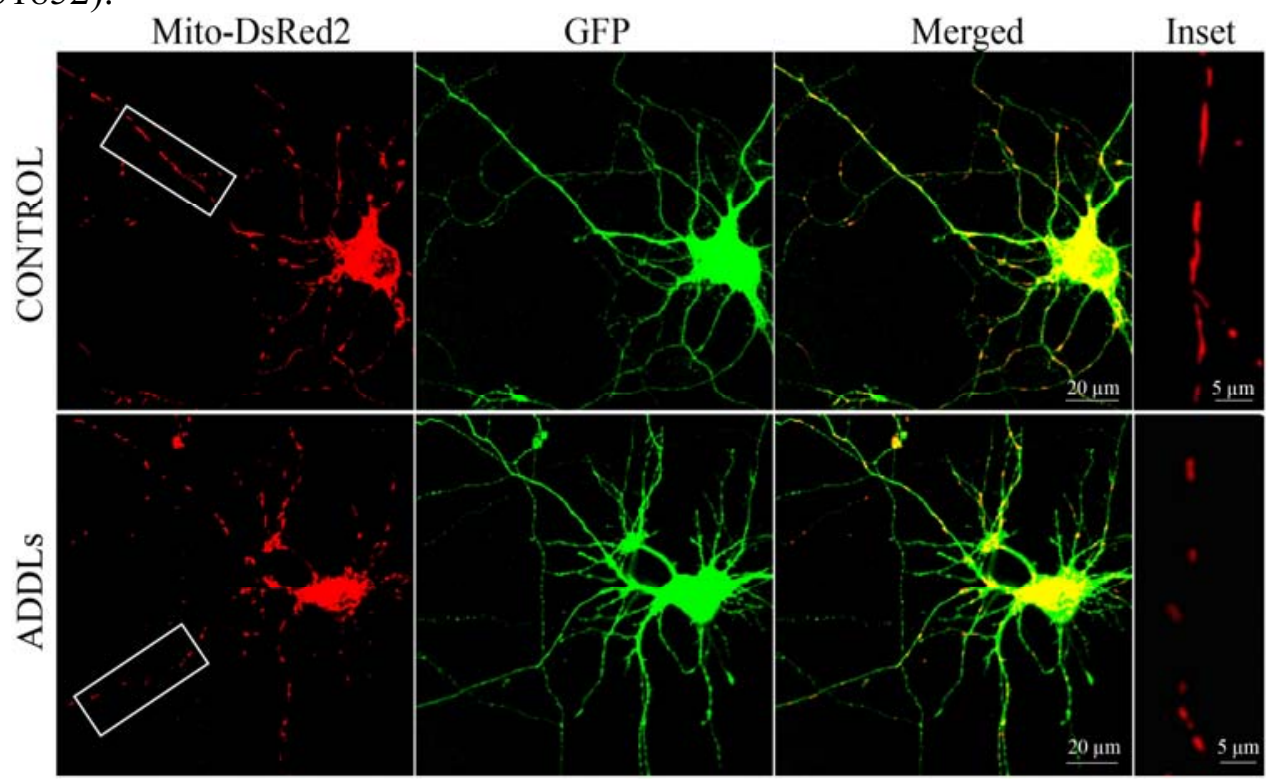

Figure 1. Effects of Amyloid $\beta$-Derived Diffusible Ligands (ADDLs) on mitochondrial in E18 rat primary hippocampal neurons. Cultured neurons (DIV10) transfected with GFP and mito-DsRed2 were treated with $800 \mathrm{nM}$ ADDLs for $24 \mathrm{~h}$. Comaring to controls, ADDLs-treated neurons demonstrated fragmented mitochondria and reduced mitochondrial coverage in axon. Green: GFP; Red: mito-DsRed2 